\title{
Wavelet-Based Frequency Response Function: Comparative Study of Input Excitation
}

\author{
K. Dziedziech, W. J. Staszewski, and T. Uhl \\ Department of Robotics and Mechatronics, Faculty of Mechanical Engineering and Robotics, AGH University of Science and Technology, \\ Al. Mickiewicza 30, 30-059 Kraków, Poland \\ Correspondence should be addressed to W. J. Staszewski; w.j.staszewski@agh.edu.pl
}

Received 12 July 2013; Accepted 5 February 2014; Published 4 June 2014

Academic Editor: Nuno Maia

Copyright (C) 2014 K. Dziedziech et al. This is an open access article distributed under the Creative Commons Attribution License, which permits unrestricted use, distribution, and reproduction in any medium, provided the original work is properly cited.

Time-variant systems can be found in many areas of engineering. It is widely accepted that the classical Fourier-based methods are not suitable for the analysis and identification of such systems. The time-variant frequency response function-based on the continuous wavelet transform-is used in this paper for the analysis of time-variant systems. The focus is on the comparative study of various broadband input excitations. The performance of the method is tested using simulated data from a simple MDOF system and experimental data from a frame-like structure.

\section{Introduction}

Vibration analysis and dynamic testing traditionally rely either on time-domain or frequency-domain approaches. However, many engineering systems exhibit time-variant behaviour. Examples include aircraft with different configurations of control surfaces during take-off and landing, deployable space structures and manipulator, tooth gear systems in mesh, or robot manipulator arms with modulator/demodulator cascade controllers. It is well known that the classical parametric and nonparametric methods are not suitable for the analysis and identification of such systems. Therefore various approaches have been developed for the analysis time-variant systems, as overviewed in [1, 2]. Wavelets are particularly attractive for the analysis and identification of time-variant systems thanks to their timefrequency (or rather time-scale) nature. Previous work in this area includes various applications in structural dynamics such as damping estimation [3-6] or nonlinear system analysis [7]. The method presented in [8] is an evolutionary approach and is also used for modal identification. Timescale approaches have been used also for online identification procedures based on adaptive wavelets [9-13]. An overview of different wavelet-based approaches can be found in [1315]. Some research work has been done to extend the classical input-output modal analysis to time-variant systems.
However, the majority of the proposed methods are adaptive approaches, developed mainly in control engineering, signal processing, and mathematics, as discussed in [1]. More recently, the time-dependent frequency response function (FRF) has been proposed [16-18] for the analysis and identification of time-variant systems. The method utilises wavelets $[16,18]$ and time-frequency transformations/distributions [17] for the calculations of time-dependent FRFs.

Since the classical, that is, Fourier-based FRF is by definition the Fourier transform of the output divided by the Fourier transform of the input, the only condition in this analysis for the input signal is that it should be Fourier-transformable. Virtually the majority of physically realisable signals satisfy this restriction. Therefore the choice of excitation signals is nearly endless. From a linear system theory point of view the estimated FRF should be independent of the nature of excitation. Also, it is important that all vibration modes are excited. Therefore a broadband excitation-such as Gaussian white nose, chirps, or impacts-are used in practice. More information on various classical excitation signals used in modal analysis can be found in $[19,20]$. There is no doubt that broadband excitation should be used also for the analysis of time-variant systems since time-variation is unknown. However, in this case the choice of excitation is no longer arbitrary as some important features could go undetected. 
This paper aims to compare various forms of broadband excitation in the time-dependent FRF used for analysis of time-variant systems. The structure of the paper is as follows. Section 2 briefly describes the continuous wavelet transform for the sake of completeness. The time-dependent, waveletbased FRF is presented in Section 3. Various broadband excitation signals used in the paper are described in Section 4. The FRF-based analysis for a simulated time-variant system is presented in Section 5. Similar analysis for an experimental system is given in Section 6. Finally the paper is concluded in Section 7.

\section{Continuous Wavelet Transform}

For the sake of completeness this section briefly describes the continuous wavelet transform. The material given in this section provides only very basic mathematical definitions with some important comments relevant to the analysis in the current investigations. More information about the method can be found in [21-23].

The continuous wavelet transform is used to divide a continuous time function into set of elementary functions called wavelets. Unlike the Fourier transform, this transform has the ability to construct a time-scale representation of a signal. This representation can be transformed into the time-frequency domain in engineering applications. One of the most desirable parameters of all time-frequency representations is a good localisation of events simultaneously in time and frequency. Other desirable properties are related to time and frequency resolutions. All these parameters are limited by the Heisenberg uncertainty principle. This principle states that the combined time-frequency resolution product has a lower bound. In other words both resolution parameters, time and frequency, cannot be arbitrarily small simultaneously. The continuous wavelet transform offers very good time-frequency localisation with variable time and frequency resolutions that depended on the so-called scale parameter. Mathematically, the CWT can be defined as

$$
X_{\omega}(a, b)=\frac{1}{\sqrt{|a|}} \int_{-\infty}^{\infty} x(t) \psi^{*}\left(\frac{t-b}{a}\right) d t
$$

where $b$ is translation indicating locality, $a$ is a scale parameter providing frequency resolution, and $\psi(t)$ is a continuous function called the mother wavelet. The normalisation $\sqrt{|a|}$ in the above equation ensures that the integral energy given by each translated and dilated wavelet is independent of the scale parameter $a$. It is important that the mother wavelet satisfies the so-called admissibility condition given by

$$
\int_{-\infty}^{\infty} \frac{|\Psi(f)|^{2}}{|f|} d f<\infty
$$

where $\Psi(f)$ is the Fourier transform of $\psi(t)$. This is required to obtain the inverse wavelet transform given by

$$
x(t)=\frac{1}{C_{\psi}} \iint_{-\infty}^{\infty} W_{\psi}(a, b) \frac{1}{\sqrt{|a|}} \psi^{*}\left(\frac{t-b}{a}\right)\left(\frac{d a d b}{a^{2}}\right) .
$$

When time-frequency localization is required from the wavelet transform, the mother wavelets must be also a window function. This additionally means that

$$
\int_{-\infty}^{\infty}|\psi(t)| d t<\infty
$$

Many different wavelet functions can be employed in the time-scale analysis. In this paper the complex Morlet wavelet function defined as

$$
\psi(t)=e^{-|t|^{2} / 2} e^{j \omega_{0} t}
$$

was used as the mother wavelet. Graphical representation of the complex Morlet wavelet in the time and frequency domain is given in Figure 1. This clearly shows limitations when the Morlet wavelet is used for time-frequency analysis. The frequency domain localisation is defined by the bandwidth of the Morlet wavelet function. As it may seem harmless in this particular example, one has to remember that, with scaling of the analysing wavelets, the ratio of frequency bandwidth to the central frequency will remain constant. Thus, when, for example, the central frequency of the analysed signal equals $50 \mathrm{~Hz}$, the wavelet transform will "see" the signal in the $40-60 \mathrm{~Hz}$ frequency range. Therefore one has to be very careful when closed vibration modes are analysed with the CWT. In practice shift and scale parameters can be selected adequately to provide correct analysis. This problem is discussed in more details in $[3,5,16]$.

\section{Wavelet-Based Frequency Response Function}

This section briefly describes the wavelet-based FRF introduced in [16-18]. The formal definition is followed by a short description of numerical implementation together with some practical comments related to averaging.

3.1. Definition. The classical FRF can be extended intuitively for time-variant systems to provide time-frequency localisation capability. When the analysis is limited to small periods of time that exhibit time-invariant behaviour, time-variations are negligibly small and the time-dependent FRF can be defined as [16-18]

$$
H(t, \omega)=\frac{Y(t, \omega)}{X(t, \omega)}=\frac{\operatorname{TF}[y(t)]}{\operatorname{TF}[x(t)]},
$$

where TF denotes the time-frequency transformation (or distribution if, for example, the Wigner-Ville transform is applied) in the above equation. The work presented in this paper utilises the continuous wavelet transform-defined in Section 2-to obtain input and output time-frequency spectra needed to calculate the time-dependent FRF. Despite the fact that (6) has a relatively simple form, the entire calculation procedure is not straightforward. It is clear that, for values of $\operatorname{TF}[x(t)]$ that are close to zero, the $H(t, \omega)$ tends to infinity. This is why additional postprocessing is required 

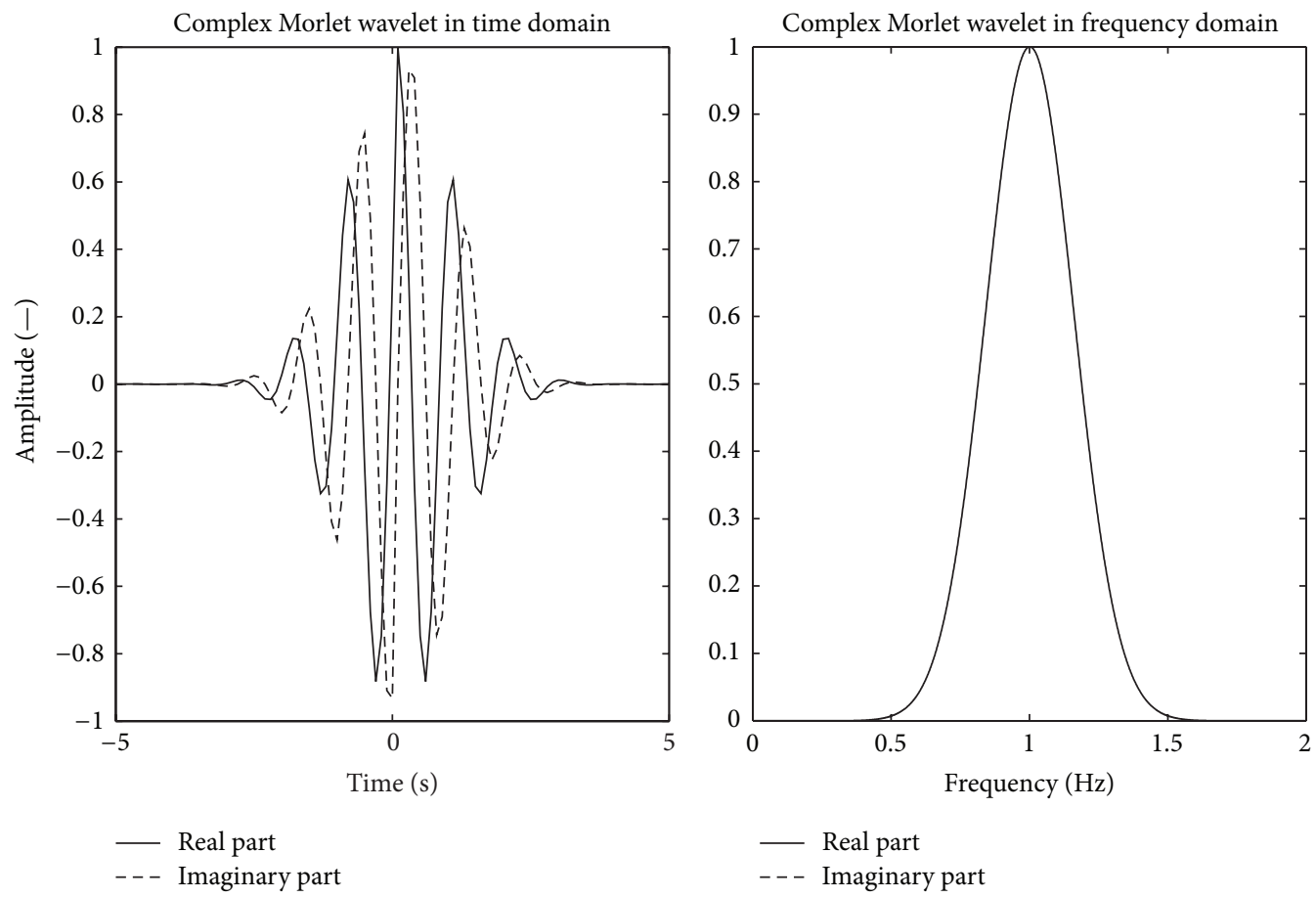

FIGURE 1: Complex Morlet wavelet function.

to avoid the above problem. In practice, the random Gaussian white noise $N$ is added to the input and output time signals to form biased (noisy) inputs $x_{i}(t)$ and outputs $y_{i}(t)$ as

$$
\begin{aligned}
& x_{i}(t)=x(t)+N_{\mu, \sigma^{2}}(t), \\
& y_{i}(t)=y(t)+N_{\mu, \sigma^{2}}(t),
\end{aligned}
$$

where $\mu$ and $\sigma^{2}$ are the mean and variance of the Gaussian distribution, respectively. Then, the relevant time-frequency spectra are calculated using the selected transformation to receive

$$
\begin{aligned}
X_{i}(t, \omega) & =\operatorname{TF}\left[x_{i}(t)\right], \\
Y_{i}(t, \omega) & =\operatorname{TF}\left[y_{i}(t)\right] .
\end{aligned}
$$

Finally, the results are averaged to obtain the expected values, that is,

$$
\begin{gathered}
E[X(t, \omega)]=\frac{1}{N} \sum_{i=1}^{N} X_{i}(t, \omega), \\
E[Y(t, \omega)]=\frac{1}{N} \sum_{i=1}^{N} Y_{i}(t, \omega)
\end{gathered}
$$

and assembled to receive the time-dependent FRF as

$$
H(t, \omega)=\frac{E[Y(t, \omega)]}{E[X(t, \omega)]} .
$$

3.2. Numerical Implementation. In practice numerical implementation is composed of several steps. Firstly, the autopower functions of input and output signals have to be defined as

$$
\begin{aligned}
G_{X X}(t, \omega) & =X(t, \omega) X^{*}(t, \omega), \\
G_{Y Y}(t, \omega) & =Y(t, \omega) Y^{*}(t, \omega),
\end{aligned}
$$

where the superscript “*” in the above equations indicated a complex conjugate. These wavelet-based autopower spectra show how the power in a signal is distributed over the entire time-frequency plane. Both functions could be also used to judge the quality of acquired input and output signals. By analogy, the so-called wavelet-based cross-power spectrum can be defined as

$$
G_{X Y}(t, \omega)=G_{Y X}(t, \omega)=X(t, \omega) Y^{*}(t, \omega) .
$$

At this point it is possible to compute the wavelet-based FRF. This can be done using different well-known estimators such as $H_{1}, H_{2}$, or $H_{v}$. In this paper the $H_{1}$ estimator based on wavelet auto- and cross-power and spectra is used as

$$
H_{1}(t, \omega)=\frac{Y(t, \omega) X^{*}(t, \omega)}{X(t, \omega) X^{*}(t, \omega)}=\frac{G_{Y X}(t, \omega)}{G_{X X}(t, \omega)} .
$$



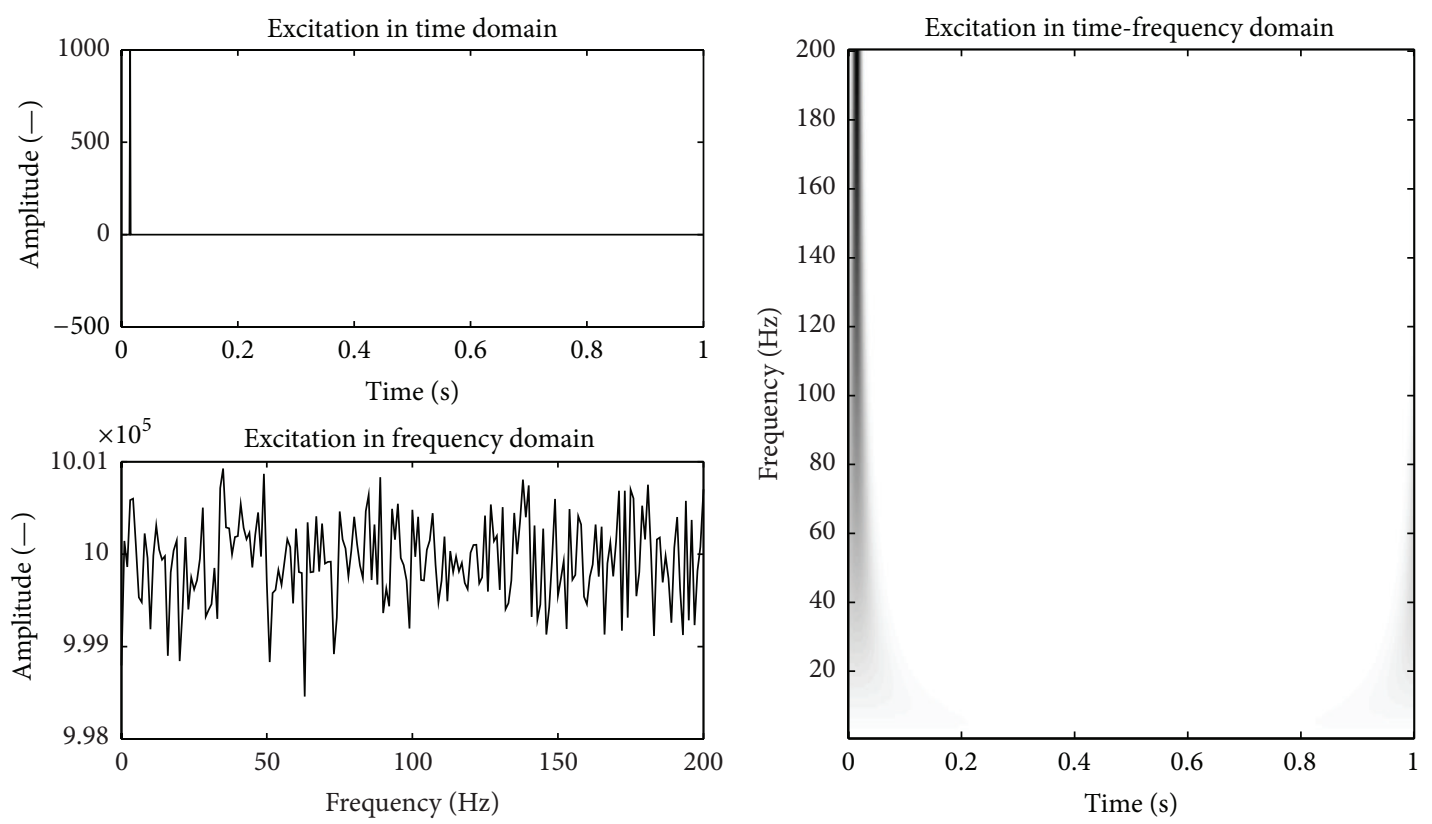

FIgURE 2: Impact excitation given in the time, frequency, and combined time-frequency domains.

In practice it is advisable to perform averaging of cross- and autopower spectra, as this improves signal-to-noise ratios leading to more reliability. Thus the relevant expected values are calculated, that is,

$$
\begin{aligned}
& E\left[G_{Y X}(t, \omega)\right]=\frac{1}{N} \sum_{i=1}^{N} G_{Y X_{i}}(t, \omega), \\
& E\left[G_{X X}(t, \omega)\right]=\frac{1}{N} \sum_{i=1}^{N} G_{X X_{i}}(t, \omega),
\end{aligned}
$$

and finally assembled to receive the wavelet-based FRF as

$$
H_{1}(t, \omega)=\frac{E\left[G_{Y X}(t, \omega)\right]}{E\left[G_{X X}(t, \omega)\right]} .
$$

Since the $H_{1}$ estimator is a complex function additional postprocessing is needed to obtain the amplitude of the wavelet-based FRF. This is simply done by calculating the absolute value of FRF; that is,

$$
H_{1_{\text {magnitude }}}(t, \omega)=\operatorname{abs}\left(H_{1}(t, \omega)\right) .
$$

This function is used to analyse time-variant systems in the following sections. The process of averaging is quite important in the entire procedure, as explained in [16]. It is also important to note that the phase can be also estimated from the wavelet-based FRF, as shown in [17]. However this characteristic is not used in the current investigations.

\section{Input Excitation for Analysis of Time-Variant Systems}

This section presents various excitation signals used in the analysis of time-variant systems. Three commonly known broadband signals and one newly proposed signal are compared in the time, frequency, and combined time-frequency domains.

Impact excitation is one of the most widely used forms of excitation in modal analysis. Figure 2 shows that the analysis of impact excitation signal in the time domain gives perfect localization in time, and the analysis in the frequency domain indicates that this excitation exhibits a truly broadband nature. These two important properties are also visible in the combined time-frequency plane. The major drawback associated with this excitation is related to the fact that the system is excited only once for a short period of time. The response is quickly damped and when (15) and (16) are used the relevant input-output wavelet-based FRF analysis will be limited only to such a short period of time.

Another commonly used excitation-which is considered to be broadband-is a chirp excitation. Figure 3 shows that the analysis of this excitation in the frequency domain indicates its broadband nature for a predefined range of frequencies. The time-domain representation of the chirp signal does not reveal immediate interesting features. However, in practice, frequency localisation could be more or less found by analysing zero-crossings and calculating relevant periods of oscillations. This indicates that a chirp is not truly broadband when only small parts of signal are considered. This behaviour is more visible in the time-frequency domain, where the analysis shows which frequency components are present at a given time.

White noise is considered to be a broadband excitation. However, this is only true when the excitation signal is long enough to be ergodic. Figure 4 shows the stationary behaviour of the Gaussian white noise excitation in the time domain. However, if one considers only a small part of this signal, the stationary and broadband behaviour is not so 

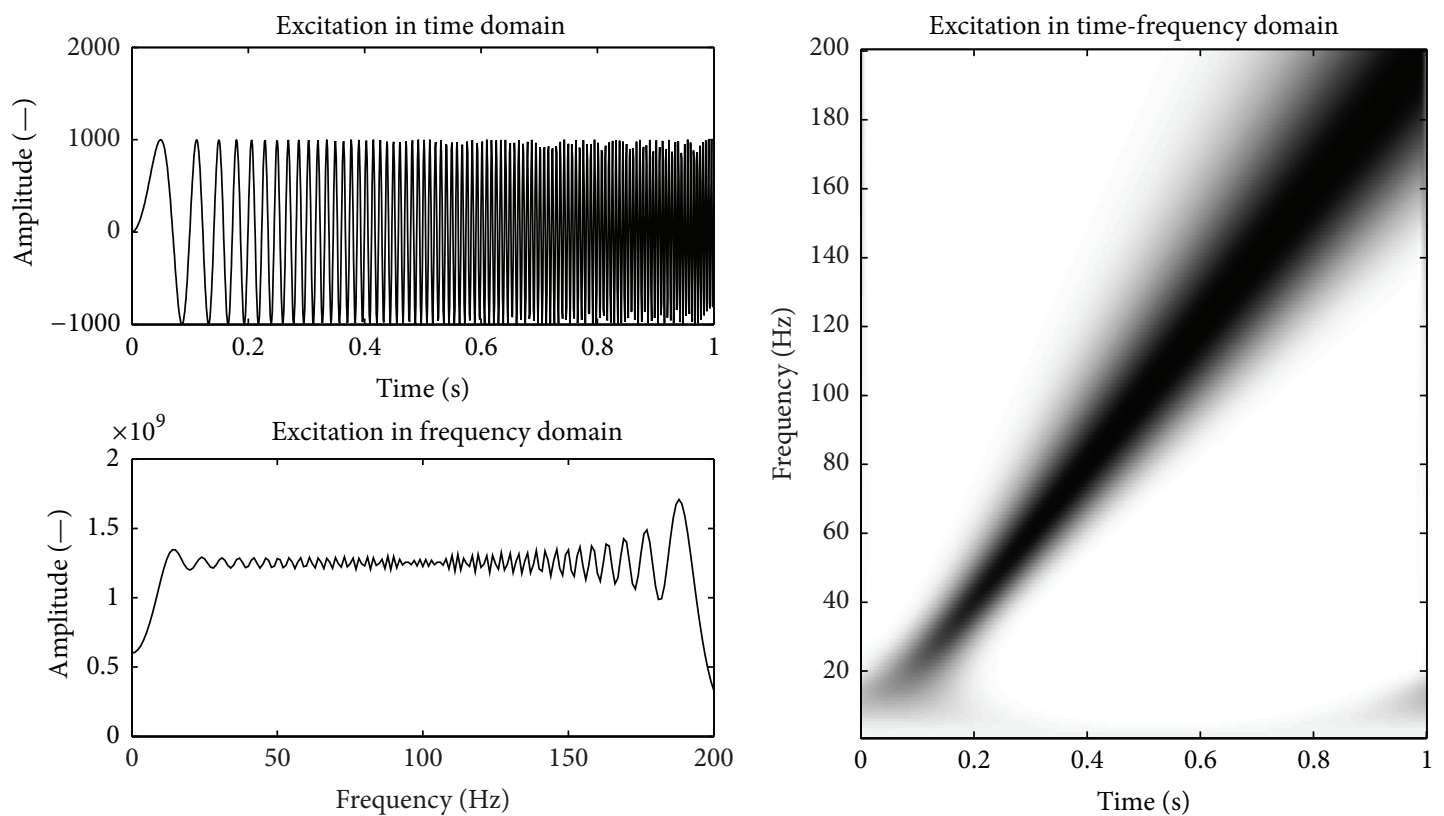

FIGURE 3: Chirp excitation given in the time, frequency, and combined time-frequency domains.
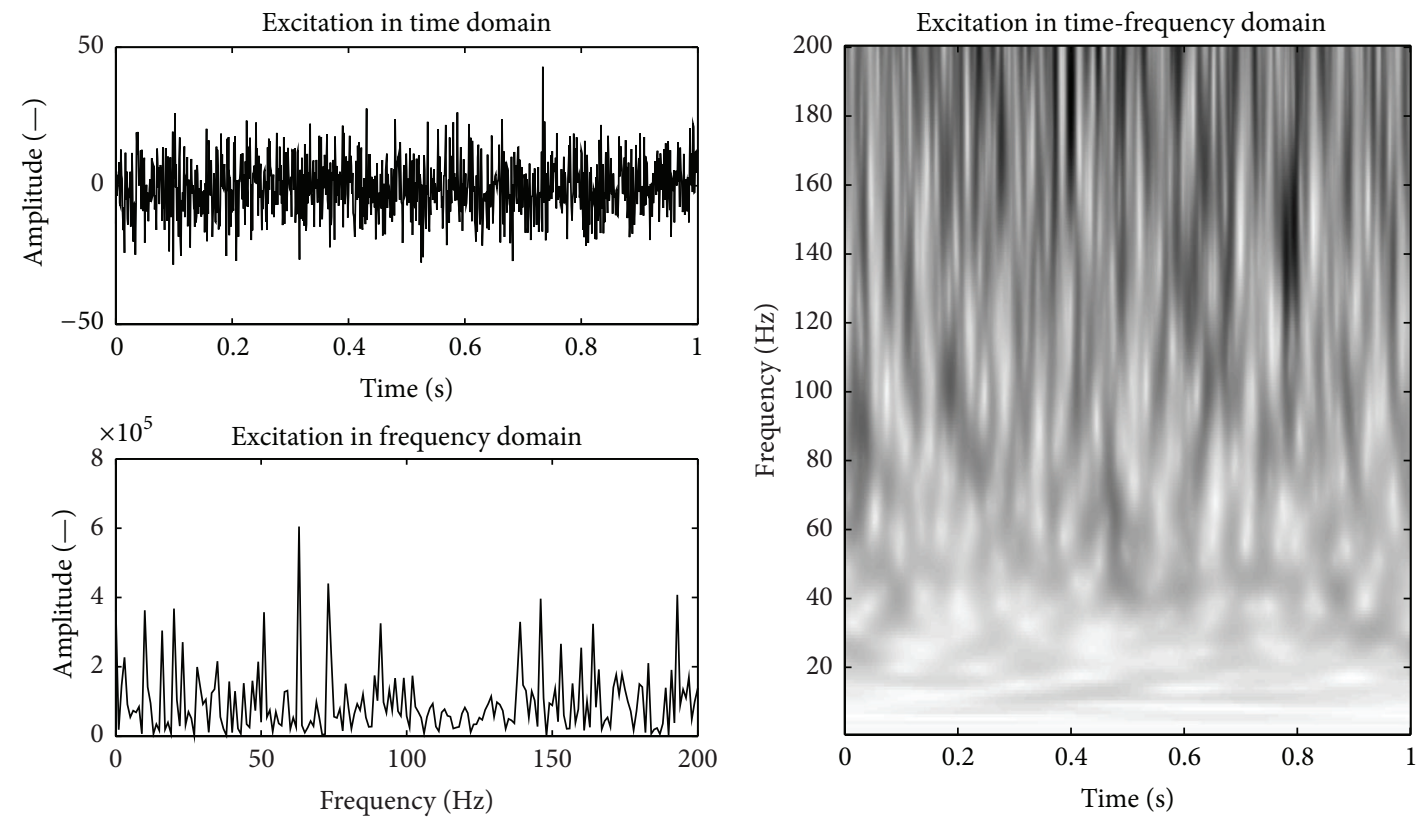

FIGURE 4: White noise excitation given in the time, frequency, and combined time-frequency domains.

obvious. This is particularly visible in the combined timefrequency domain where the energy distribution of the white noise signal varies with time and frequency from high to near zero amplitudes.

A new type of broadband excitation is proposed in this section. The proposed excitation is composed of randomly distributed series of impacts. Such excitation is broadband at given time instances and provides continuous inflow of energy to the analysed system, as illustrated in Figure 5. This is advantageous when the excitation is compared with the classical impact excitation illustrated in Figure 2. The continuous inflow of energy enables one to observe system dynamic properties and their variations with time.

\section{Numerical Simulations and Results}

5.1. Simulated MDOF System. A simple two-degree-of-freedom (2-DOF) system was used to compare the performance of input excitation signals in the analysis of time-variant systems. The system consisted of heavily damped mass-spring 

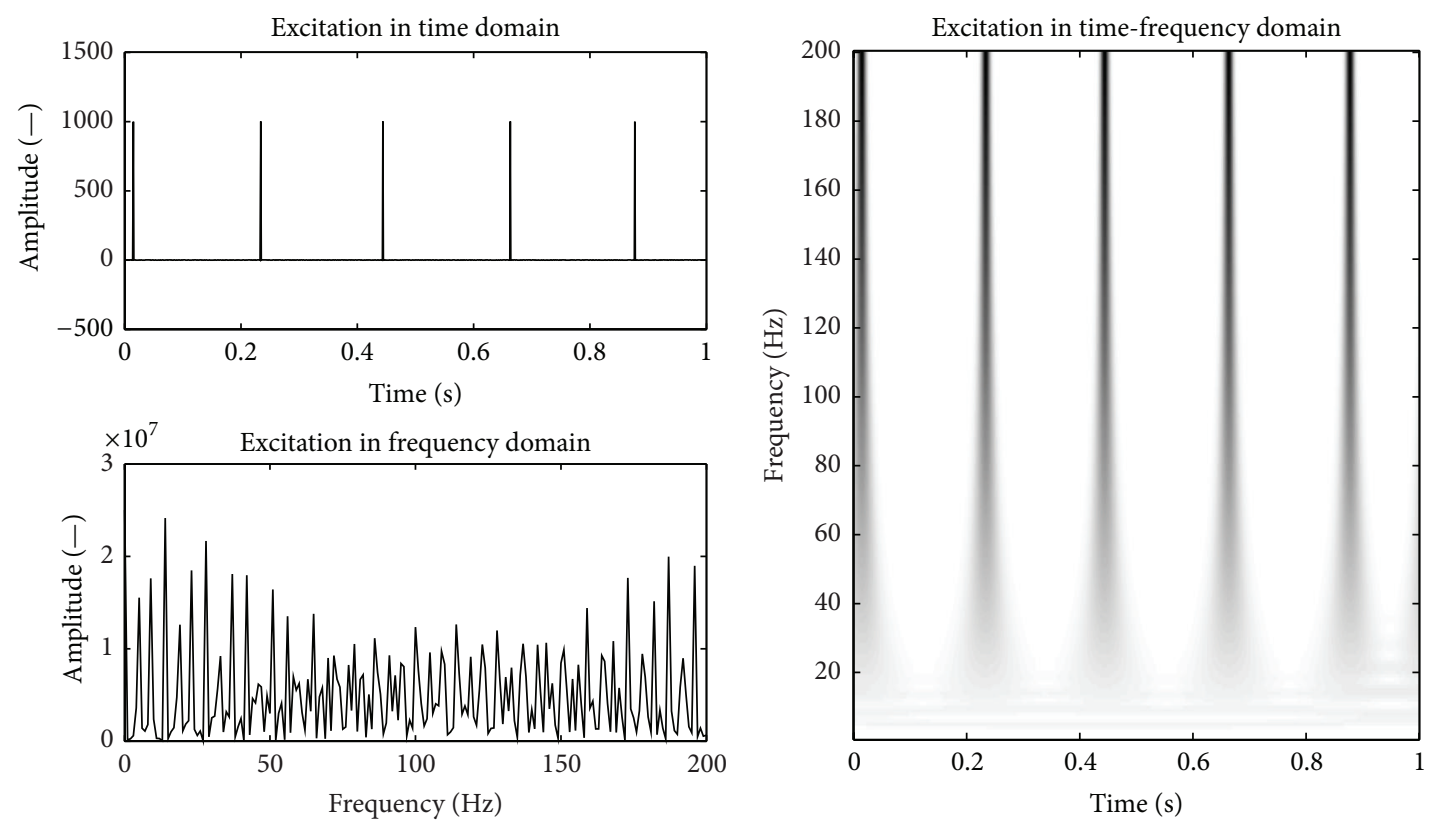

FIGURE 5: Random impact excitation in the time, frequency, and combined time-frequency domains.

elements. One of the masses involved was varying in time; the mass was decreasing linearly with time. All other relevant physical parameters were constant. The values of physical parameters used were $m=10 \mathrm{~kg}, K_{1}=3000000 \mathrm{~N} / \mathrm{m}, K_{2}=$ $800000 \mathrm{~N} / \mathrm{m}, C_{1}=150 \mathrm{~N} /(\mathrm{m} / \mathrm{s})$, and $C_{2}=350 \mathrm{~N} /(\mathrm{m} / \mathrm{s})$. The $M_{1}$ mass element was decreasing linearly in time following the function $M_{1}(t)=16-12 t \mathrm{~kg}$. Clearly, this resulted in the increment of the relevant natural frequencies. Theoretical natural frequencies are equal to $40 \mathrm{~Hz}$ and $80 \mathrm{~Hz}$ (for $0 \mathrm{~s}$ ) increasing to $160 \mathrm{~Hz}$ (for $1 \mathrm{~s}$ ) with cubic dependence on time. Figure 6 gives a schematic diagram of the simulated system. Numerical simulations were performed using the MATLAB/Simulink platform. The sampling frequency was equal to $1 \mathrm{kHz}$ in all simulations.

5.2. System Identification Results. This section describes system identification results for the simulated time-variant system described in Section 5.1. The focus is on the analysis of varying natural frequencies. Numerical calculations were performed within the MATLAB platform, following the procedure described in Section 3. Signal averaging was used in order to smooth out different disturbances that had impact on the amplitude of the wavelet-based FRF. Four different excitations-described in Section 4-were tested. The classical FRF was also computed for comparison.

Figure 7 shows the results for the impact excitation. The response of the system in the time domain reveals heavy damping in the simulated system; the envelope amplitude decays relatively quickly. The classical FRF clearly exhibits two vibration modes. The first natural frequency was found to be around $39 \mathrm{~Hz}$ whereas the second natural frequency could be estimated around $83 \mathrm{~Hz}$. There is very little indication about possible time-variant behaviour, as expected. The second FRF component is more wideband and slightly skewed

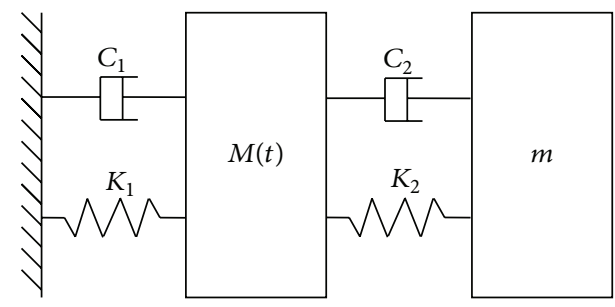

FIGURE 6: Schematic diagram of the 2-DOF system used in numerical simulations.

(or asymmetrical). This could indicate some nonlinearity and/or time-variance. Also, it is very difficult to observe any time-variant behaviour in the amplitude contour plot of the wavelet-based FRF. Harmonic oscillations are dampened too quickly. Some indication on the varying nature is visible in the second vibration mode.

The second considered input signal was a chirp excitation. The results for this excitation are presented in Figure 8. This excitation is renowned for its frequency evolution over time. Therefore both vibration modes are excited not earlier than the chirp sweeps through the relevant natural frequencies. The first natural frequency was found to be around $39 \mathrm{~Hz}$, whereas the second natural frequency was estimated around $104 \mathrm{~Hz}$. The amplitude of the wavelet-based FRF does not indicate any time-varying nature of the system.

White noise excitation differs qualitatively from impact and chirp excitation as it provides energy continuously through the entire excitation time. However, amplitude excitation levels vary for given frequencies. Thanks to this property it is possible to observe time-varying nature of the analysed mechanical system in Figure 9. Although the classical FRF is not clear, two vibration modes can be observed in 

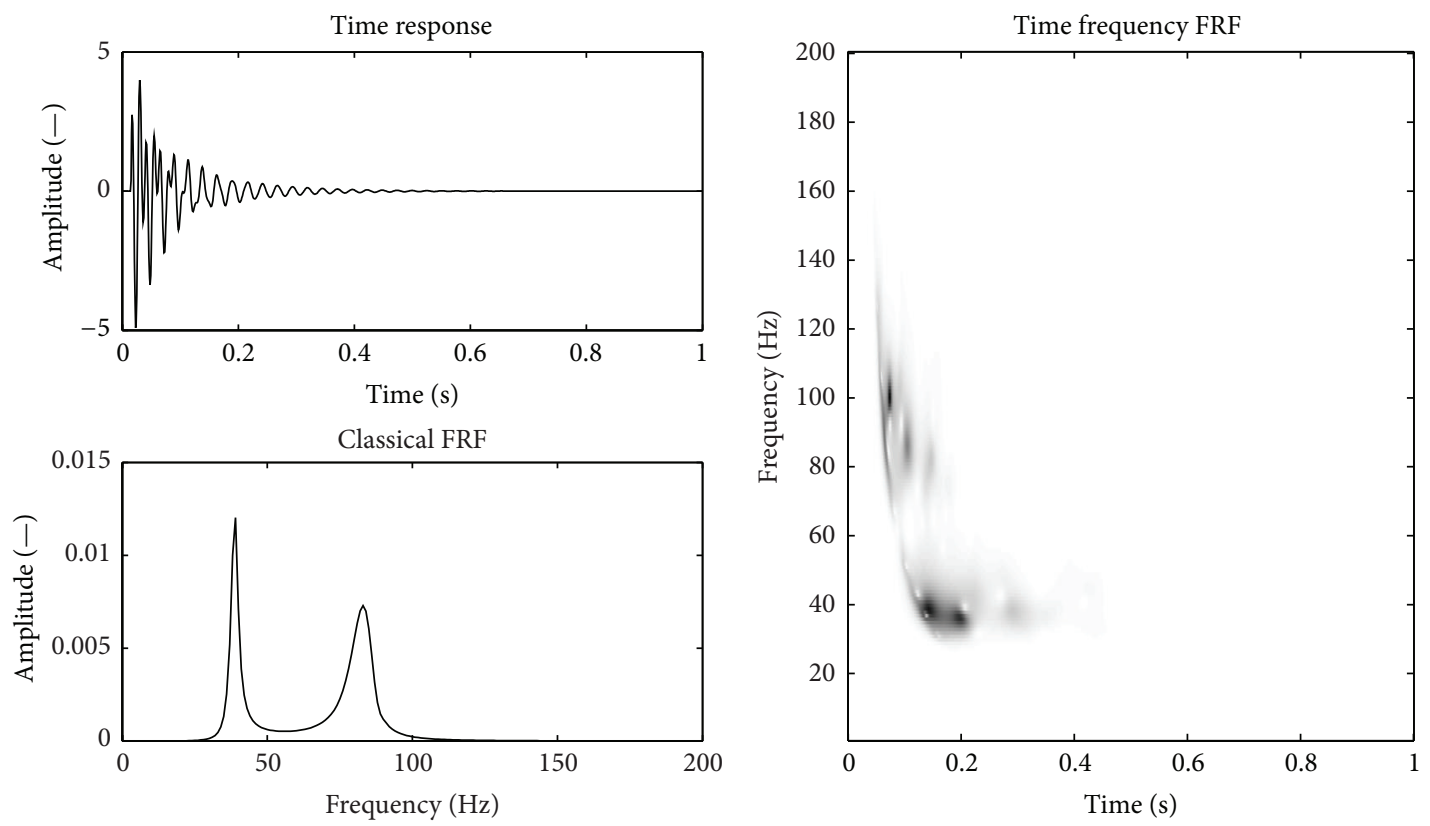

FIGURE 7: Simulated results obtained for the impact excitation in the time, frequency, and combined time-frequency domains.
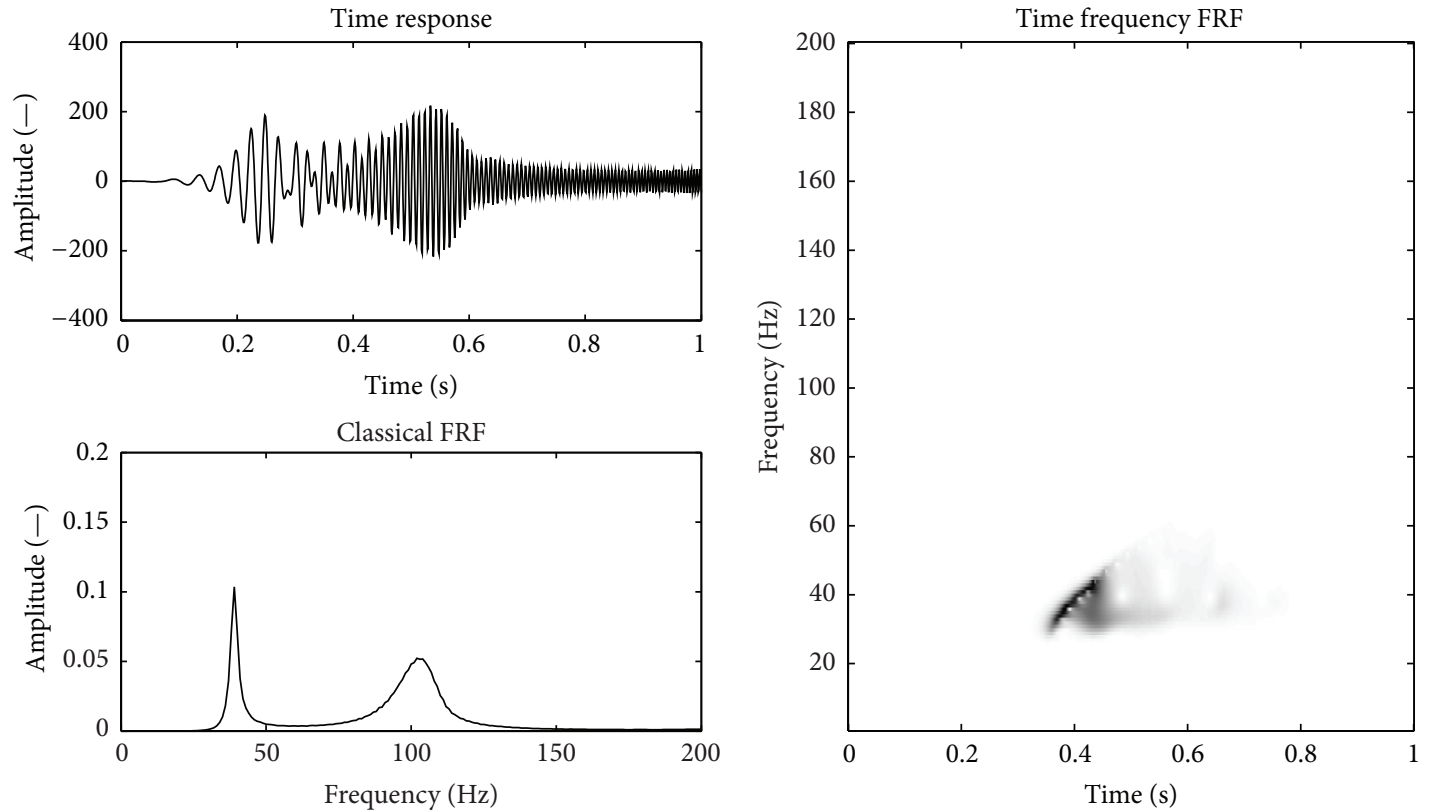

FIGURE 8: Simulated results obtained for the chirp excitation in the time, frequency, and combined time-frequency domains.

the amplitude of the wavelet-based FRF. The latter can be used to estimate the natural frequencies. The first natural frequency was found to be around $39 \mathrm{~Hz}$ whereas the second natural frequency was found to vary between 80 and $150 \mathrm{~Hz}$. The only problem is that the curves displaying vibration modes in the amplitude of the wavelet-based FRF are broken and do not show uniform energy distribution.

The random impact excitation consists of repeated impacts in order to introduce more energy into the system. The amplitude of impacts was constant whereas the repletion time was random in this type of excitation. The results are presented in Figure 10. The time-domain response of the system exhibits five repeated impact responses. However, periods of these impacts are different; that is, the frequency involved increases. The classical FRF displays correctly only the first vibration mode. The time-variant nature can be clearly observed in the amplitude contour plot of the waveletbased FRF. The first natural frequency was found to be around $39 \mathrm{~Hz}$ whereas the second natural frequency was estimated to vary between 80 and $150 \mathrm{~Hz}$. Similarly to the white noise 

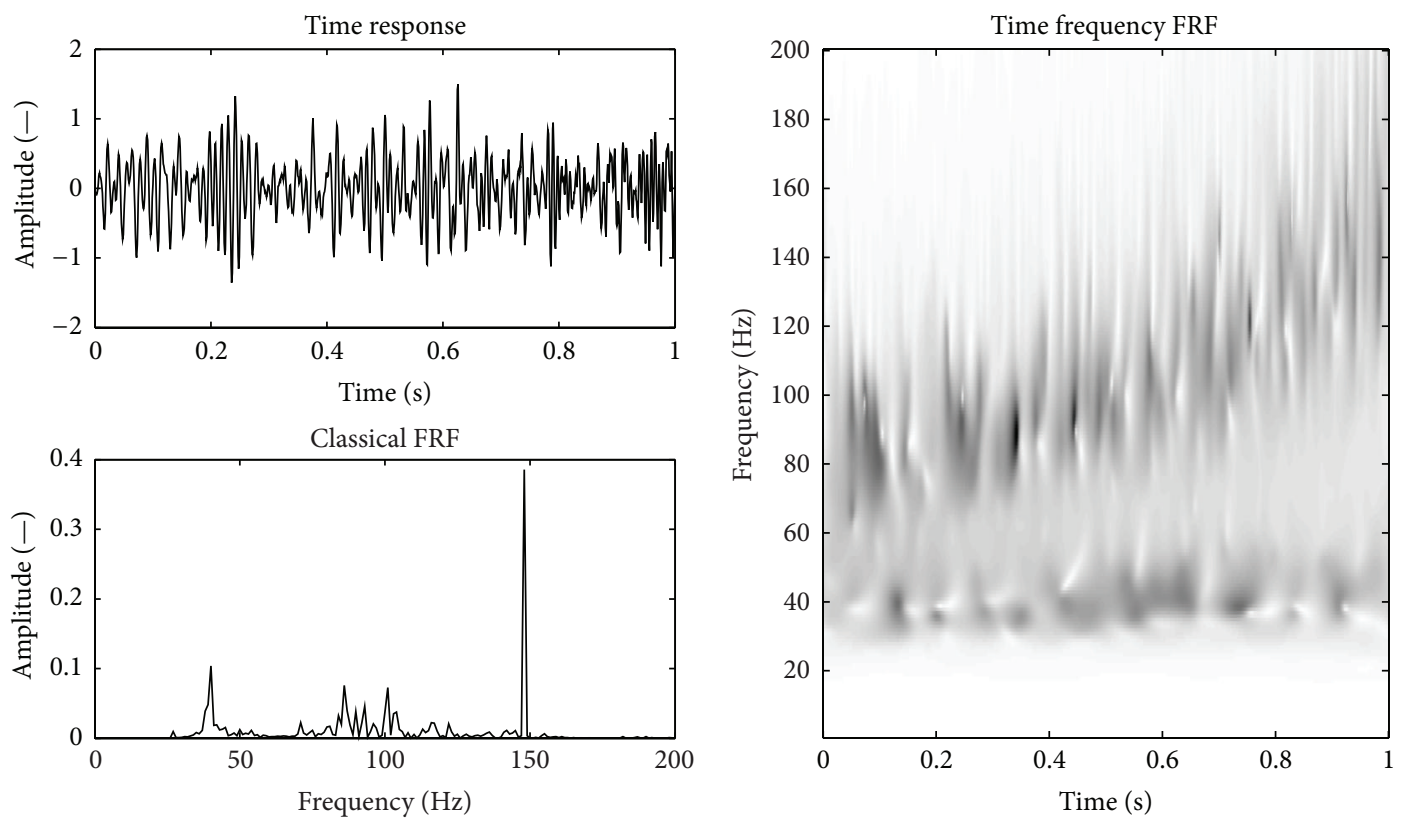

FIGURE 9: Simulated results obtained for the white noise excitation in the time, frequency, and combined time-frequency domains.
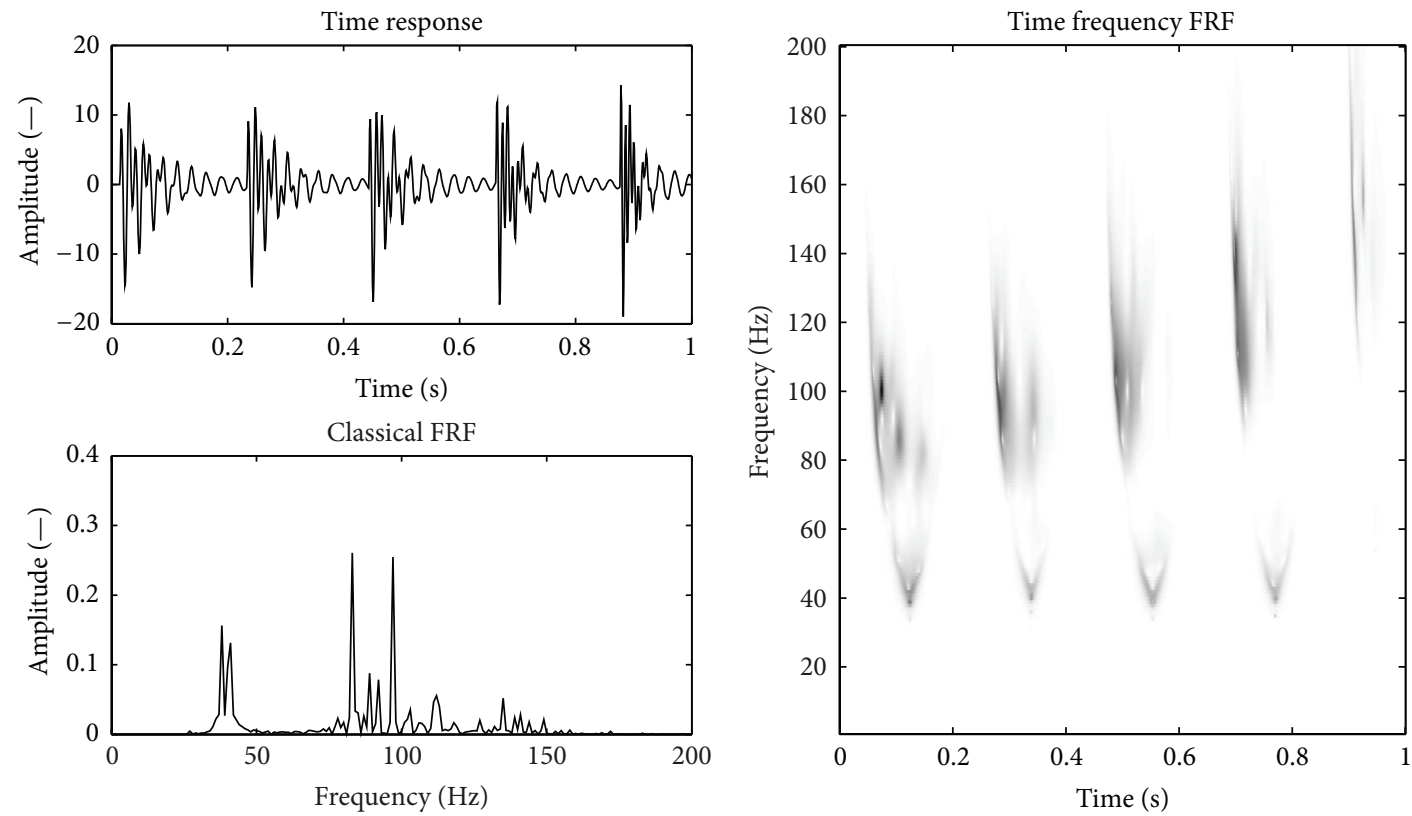

FIGURE 10: Simulated results obtained for the repeated impact excitation in the time, frequency, and combined time-frequency domains.

excitation, the curves displaying vibration modes in the amplitude of the wavelet-based FRF are broken. However this time the energy distribution for all impacts is more uniform.

In summary, the noise and repeated impact input signals were the only out of the four excitations tested that were able to reveal time-variant nature of the analysed system in the amplitude of the wavelet-based FRF. Therefore these two excitations were further tested using the experimental data, as described in the next section.

\section{Experimental Application and Results}

A simple experiment was conducted to obtain time-variant vibration data. A frame-like structure-shown in Figure 11with a tank attached to the free end was used in this experimental work. The vertical arm was $80 \mathrm{~cm}$ long, whereas the horizontal arm was $60 \mathrm{~cm}$ long. The tank was filled with $3 \mathrm{~kg}$ of sand. The tank was draining when vibration measurements were taken using one accelerometer mounted 


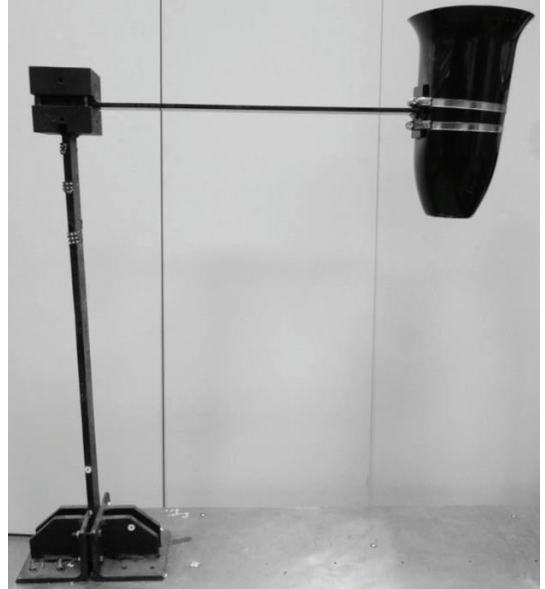

FIGURE 11: Experimental time-variant test rig.

in the middle of the vertical arm of frame. Accelerometer was measuring vibrations in horizontal direction. Varying mass of the tank contributed to time-varying behaviour of the entire system. The system was excited with a modal hammer and electrodynamic shaker. The middle of metal block joining horizontal and vertical arms was used as excitation point. Excitation was executed in horizontal direction. Due to the time-varying nature of analysed phenomena, identical multiple measurements-required for averaging-were not possible. Therefore Gaussian white noise was used to corrupt the data. The relevant responses were then calculated using (7). In order to improve the visibility of the results, the entire analysis was limited only to the frequency range from 230 to $300 \mathrm{~Hz}$. The amplitudes of wavelet-based FRFs were calculated. Contour plots together with ridges were used for the analysis of the system. The latter exhibits amplitude where the largest energies are concentrated. These areas correspond to vibration modes. These ridges were calculated following the procedure described in [24].

The results for the noise excitation are shown in Figure 12. Two vibration modes can be observed in the analysed frequency range. The first mode can be seen for the entire period of time, that is, between 0 and $80 \mathrm{~s}$. Its frequency varies from the initial value of $276 \mathrm{~Hz}$ (for $0 \mathrm{~s}$ ) to the final value of $256 \mathrm{~Hz}$ (for $80 \mathrm{~s}$ ). After $5 \mathrm{~s}$ the second mode appears. The frequency of this mode remains relatively unchanged and can be estimated as approximately equal to $290 \mathrm{~Hz}$. The results for the repeated impact excitation are given in Figure 13. These results reveal almost the same time-variant behaviour. Only one mode can be observed initially. Its frequency varies from 275 to $259 \mathrm{~Hz}$. Then this mode splits into two modes. The frequency of the new (i.e., second) mode again remains relatively unchanged and can be estimated as $290 \mathrm{~Hz}$; this mode was not visible until 25 s probably due to high damping of the structure. The response amplitude decayed very fast when the impact excitation was used. That was not the case when the white noise excitation was applied. In summary, both types of excitation used, that is, the Gaussian white noise and random repeated impacts, have revealed correctly the varying nature of the analysed system. One important

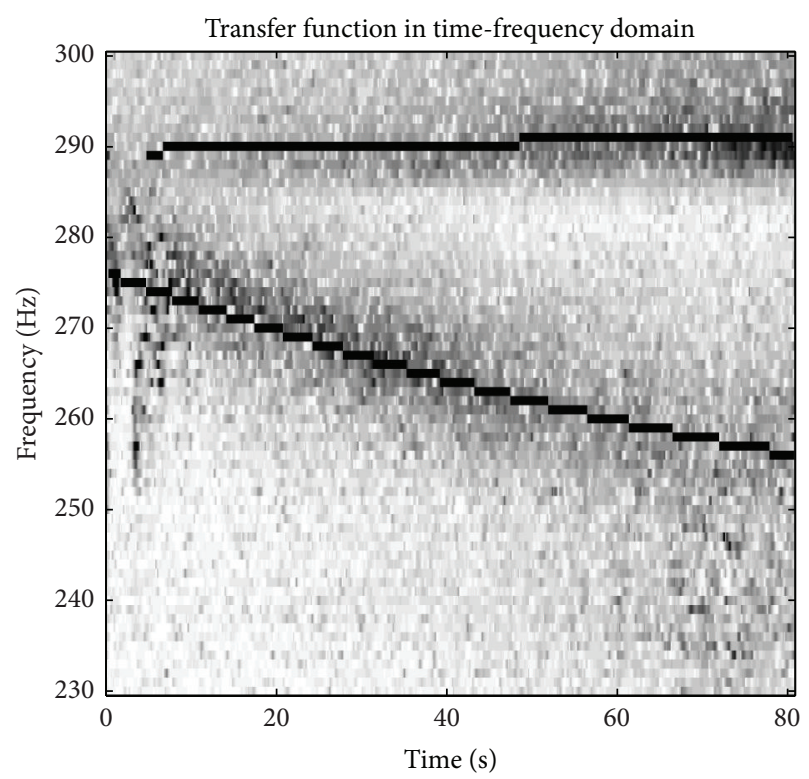

Figure 12: Experimental results obtained for the white noise excitation in the combined time-frequency domain.

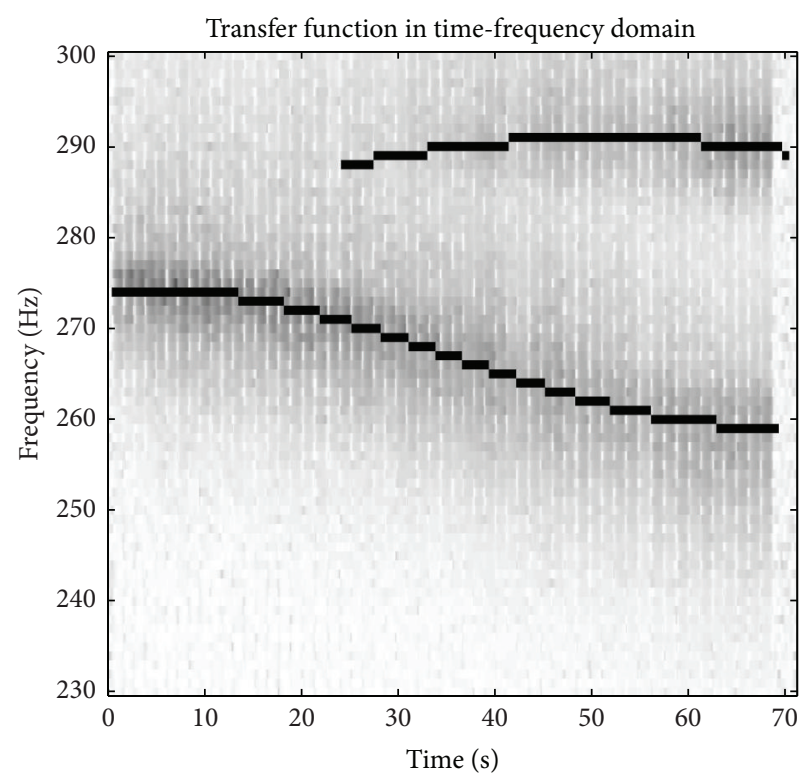

FIGURE 13: Experimental results obtained for the repeated impact excitation in combined time-frequency domain.

feature can be observed when Figures 12 and 13 are compared. The natural frequency of the first analysed mode shifts downwards more rapidly, that is, from 276 to $256 \mathrm{~Hz}$, when the noise excitation was used in Figure 12.

An additional comment is needed here regarding the change of frequency when mass of structure decreased. It is well known that, for a simple 1-DOF system, the decrease of mass results in the increase of natural frequency. However, this basic theoretical relation does not need to hold for complex MDOF systems, especially for systems where the major mass is concentrated in one part of the structure. Although 
this is a common problem found in experimental modal analysis, the observed effect needs further investigations.

\section{Conclusions}

The wavelet-based FRF was used for the analysis of timevariant systems. The performance of the method was compared for four different types of broadband excitation. Numerical simulations and experimental test data were used in these investigations. A simple heavily dampened 2-DOF system with a time-varying mass was simulated. A frame-like structure with an attached tank was used for experimental tests.

The results show that-for the case investigated-it is impossible to identify time-varying nature of system with the use of impact and chirp excitation. These two excitations do not excite the system continuously in a proper broadband manner. Identification of time-varying systems was possible with the use of two other excitations applied, that is, the white noise and random impact excitations. Both excitations correctly identified time-varying nature of the systems investigated.

It is important to note that the effect of excitation signals was investigated only for the amplitude of the waveletbased FRF. Future work should involve the analysis of FRF's phase and other modal parameters (than natural frequency). Furthermore, more complex experimental systems should be investigated.

\section{Conflict of Interests}

The authors declare that there is no conflict of interests regarding the publication of this paper.

\section{Acknowledgment}

The work presented in this paper was supported by funding from the Research Project WELCOME (Foundation for Polish Science, Project no. 2010-3/2).

\section{References}

[1] A. G. Poulimenos and S. D. Fassois, "Parametric time-domain methods for non-stationary random vibration modelling and analysis-a critical survey and comparison," Mechanical Systems and Signal Processing, vol. 20, no. 4, pp. 763-816, 2006.

[2] M. D. Spiridonakos and S. D. Fassois, "Parametric identification of a time-varying structure based on vector vibration response measurements," Mechanical Systems and Signal Processing, vol. 23, no. 6, pp. 2029-2048, 2009.

[3] W. J. Staszewski, "Identification of damping in MDOF systems using time-scale decomposition," Journal of Sound and Vibration, vol. 203, no. 2, pp. 283-305, 1997.

[4] W. J. Staszewski and J. E. Cooper, "Wavelet approach to flutter data analysis," Journal of Aircraft, vol. 39, no. 1, pp. 125-132, 2002.

[5] T. P. Le and P. Argoul, "Continuous wavelet transform for modal identification using free decay response," Journal of Sound and Vibration, vol. 277, no. 1-2, pp. 73-100, 2004.
[6] S. L. Chen, J. J. Liu, and H. C. Lai, "Wavelet analysis for identification of damping ratios and natural frequencies," Journal of Sound and Vibration, vol. 323, no. 1-2, pp. 130-147, 2009.

[7] W. J. Staszewski, "Identification of non-linear systems using multi-scale ridges and skeletons of the wavelet transform," Journal of Sound and Vibration, vol. 214, no. 4, pp. 639-658, 1998.

[8] P. Tratskas and P. D. Spanos, "Linear multi-degree-of-freedom system stochastic response by using the harmonic wavelet transform," Journal of Applied Mechanics, Transactions ASME, vol. 70, no. 5, pp. 724-731, 2003.

[9] T. Uhl, M. Petko, G. Karpiel, and A. Klepka, "Real time estimation of modal parameters and their quality assessment," Shock and Vibration, vol. 15, no. 3-4, pp. 299-306, 2008.

[10] A. Klepka and T. Uhl, "Hardware and software tools for inflight flutter testing," in Proceedings of the ISMA International Conference on Noise and Vibration Engineering, vol. 1-8, pp. 1207-1221, 2008.

[11] R. Zouari, L. Mevel, A. Klepka, and M. Basseville, "Adaptive flutter monitoring using wavelet filtering and recursive subspacebased detection," in Proceedings of the 4th European Workshop on Structural Health Monitoring, pp. 1137-1144, Krakow, Poland, July 2008.

[12] B. Basu, S. Nagarajaiah, and A. Chakraborty, "Online identification of linear time-varying stiffness of structural systems by wavelet analysis," Structural Health Monitoring, vol. 7, no. 1, pp. 21-36, 2008.

[13] S. Nagarajaiah and B. Basu, "Output only modal identification and structural damage detection using time frequency \& wavelet techniques," Earthquake Engineering and Engineering Vibration, vol. 8, no. 4, pp. 583-605, 2009.

[14] W. J. Staszewski and A. N. Robertson, "Time-frequency and time-scale analyses for structural health monitoring," Philosophical Transactions of the Royal Society A, vol. 365, no. 1851, pp. 449-477, 2007.

[15] A. N. Roberston and B. Basu, "Wavelet analysis," in Encyclopedia on Structural Health Monitoring, C. Boller, F. K. Chang, and Y. Fujino, Eds., John Wiley \& Sons, 2009.

[16] W. J. Staszewski and D. M. Wallace, "Wavelet-based frequency response function for time-variant systems-an exploratory study," Mechanical Systems and Signal Processing, vol. 47, no. 1-2, 2014.

[17] K. Dziedziech, W. J. Staszewski, and T. Uhl, "Input-output timefrequency analysis of time-variant systems," in Proceedings of the International conference on Uncertainty in Structural Dynamics, pp. 2765-2774, Leuven, Belgium, September 2012.

[18] W. J. Staszewski and J. Giacomin, "Application of the wavelet based FRFs to the analysis of nonstationary vehicle data," in 15th International Modal Analysis Conference, vol. 3089 of Proceedings of SPIE, pp. 425-431, The International Society for Optical Engineering, 1997.

[19] D. Brown, K. Ramsey, and G. Carbon, Survey of Excitation Techniques Applicable to the Testing of Automotive Structures, SAE Technical Paper 770029, 1977.

[20] J. Schoukens, R. Pintelon, E. van der Ouderaa, and J. Renneboog, "Survey of excitation signals for FFT based signal analyzers," IEEE Transactions on Instrumentation and Measurement, vol. 37, no. 3, pp. 342-352, 1988.

[21] L. Cohen, Time Frequency Analysis: Theory and Applications, Prentice Hall, New York, NY, USA, 1st edition, 1994. 
[22] R. Carmona, W. L. Hwang, and B. Torrésani, Practical TimeFrequency Analysis: Gabor and Wavelet Transforms with an Implementation in S, Academic Press, New York, NY, USA, 1998.

[23] S. Mallat, A Wavelet Tour of Signal Processing: The Sparse Way, Academic Press, New York, NY, USA, 3rd edition, 2008.

[24] K. Dziedziech, W. J. Staszewski, and T. Uhl, "Wavelet-based modal analysis for time-variant systems," Mechanical Systems and Signal Processing. In press. 

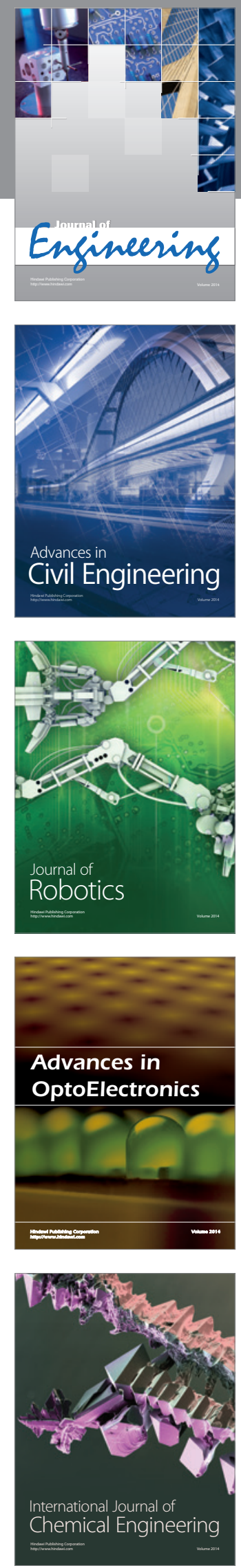

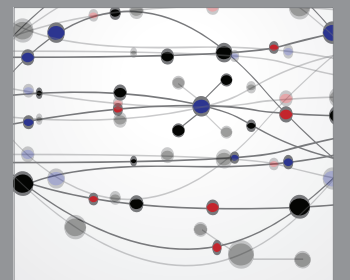

The Scientific World Journal
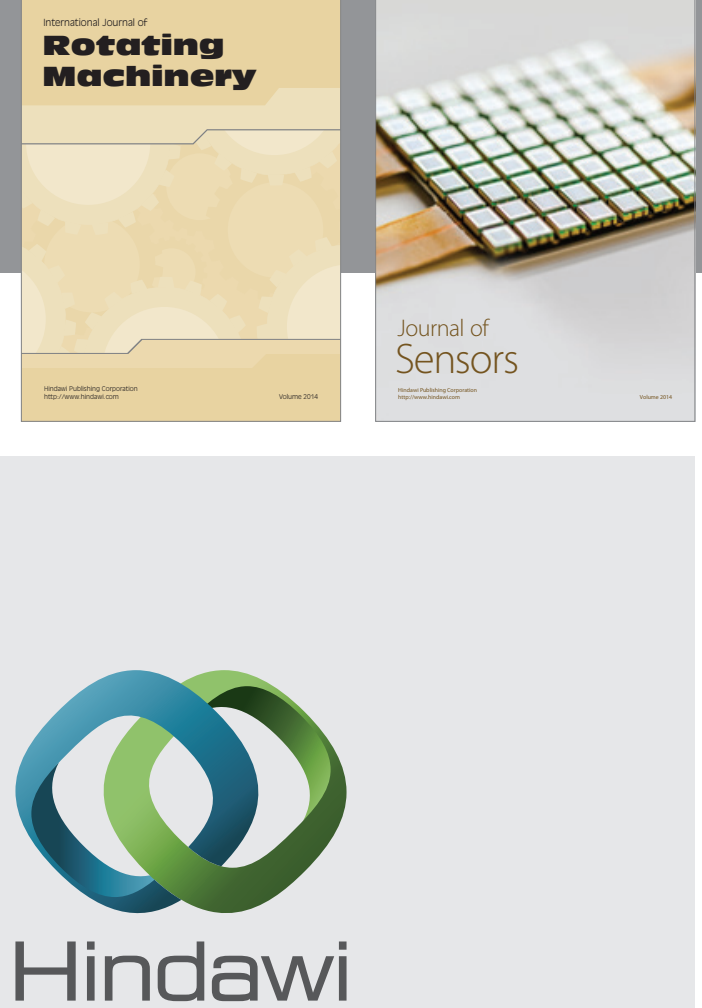

Submit your manuscripts at http://www.hindawi.com
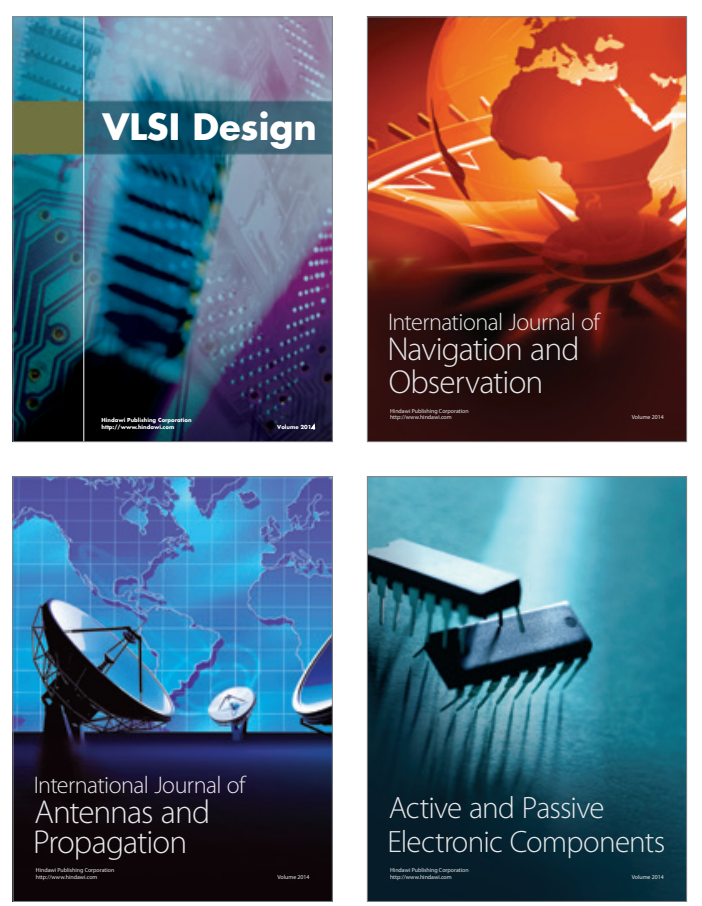
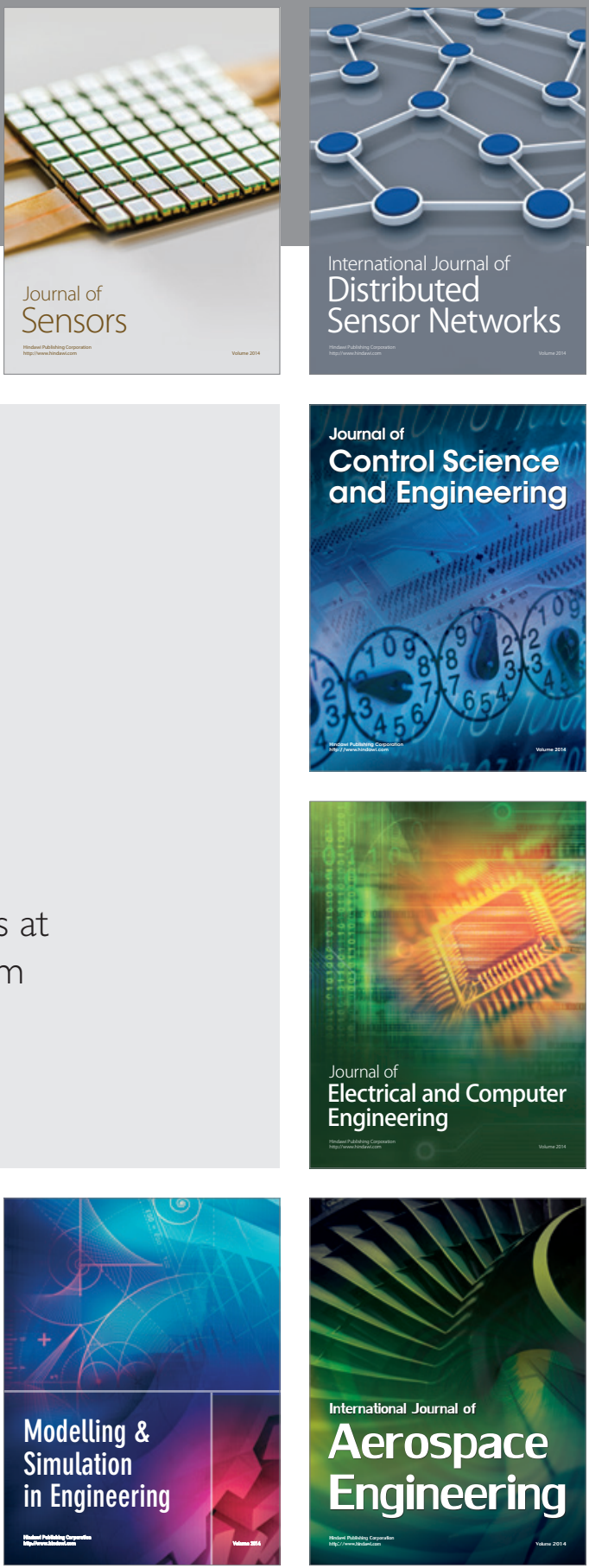

Journal of

Control Science

and Engineering
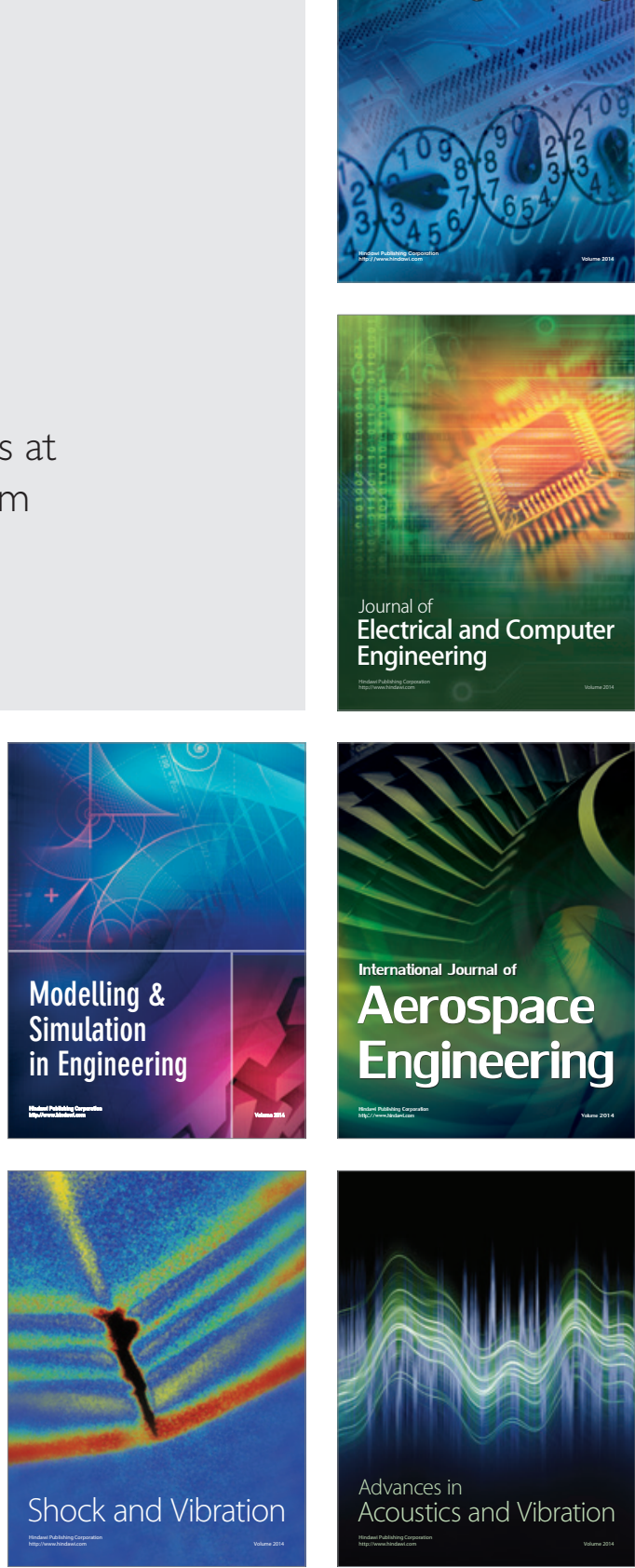